\section{Endoscopic Diagnosis and Treatment of an Esophageal Bezoar Resulting from Bulk Laxative Ingestion}

The finding of a bezoar during gastrointestinal endoscopy is not frequent, and the location is generally in the stomach $(1,2)$, although it has been described as an occasional finding in the esophagus (3-5). The material of the bezoar can vary, but it is generally composed of hair or dietary nonabsorbable fiber. We present here the case of a 66-year-old man with a history of chronic constipation, who had been treated with a bulk laxative consisting of plantago ovata seeds in granular form. The patient was referred to our service with acute dysphagia and retrosternal pain. The patient's previous medical history was essentially unremarkable, except for an episode of esophagitis diagnosed as hiatal hernia with minimal gastroesophageal reflux that had been treated with ranitidine for the previous two years. The physical examination was normal.

During endoscopy, a localized mass in the distal third of the esophagus was visualized, completely occluding the lumen (Figure 1). This corresponded to a bezoar that was fragmented into multiple pieces and partially extracted with the help of a polypectomy snare (Figure 2), allowing the endoscope to be advanced into the stomach along with some fragments of the bezoar.

To our knowledge, only five cases of esophageal bezoars have been previously described (3-5). Although the incidence of this complication is rare, the increasing use of bulk laxatives may favor the development of this disorder.

J. Pérez-Piqueras, C. Silva, J. Jaqueti, M.A. Sáez,

D. Martinez, J. M. Santa, I. Peralba

Hospital Universitario del Aire, Madrid, Spain

References

1. Hermoso JC, Rosado R, Ramirez D et al.: Postsurgical gastric bezoar: presentation of a case of esophageal impaction. Rev Esp Enferm Dig 1991; 79: 139-141.

2. Davion T, Delamarre $J$, Reix $N$ et al.: Gastric bezoar: another side effect of endoscopic variceal sclerotherapy. Scand J Gastroenterol 1989: 24: $818-820$

3. Schneider RP: Perdiem causes esophageal impaction and bezoars South Med J 1989; 82: 1449-1450.

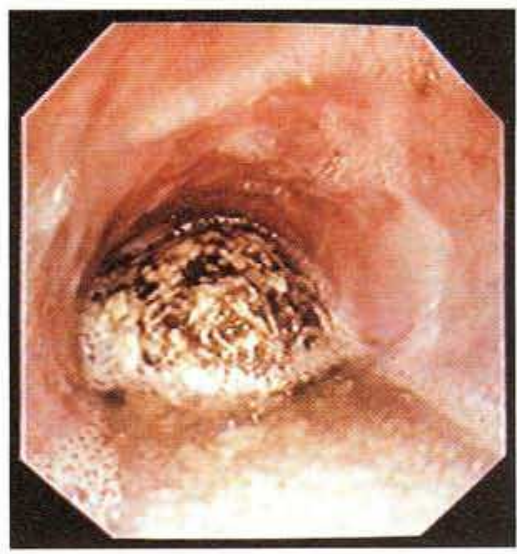

Figure 1:

A bezoar with a brownish color and granular aspect occluding the lumen of the esophagus.

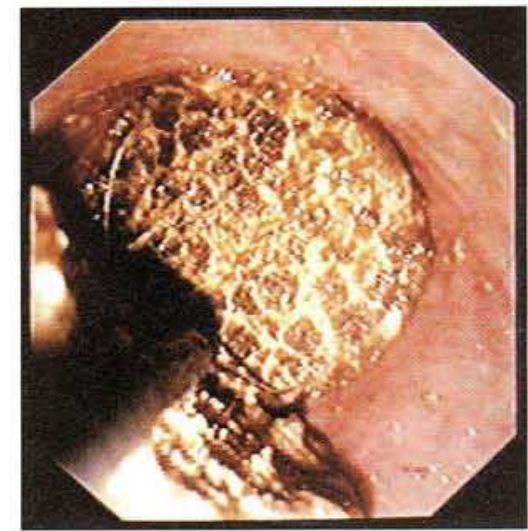

Figure 2:

Endoscopic view of the manipulation and removal of the bezoar using the polypectomy snare.

4. Anderson W. Weatherstone $G$, Veal $C$ : Esophageal medication bezoar in a patient receiving enteral feedings and sucralfate. Am J Gastroenterol 1989; 84: 205-206.

5. Shueke M, Mihas AA: Esophageal bezoar due to sucralfate. Endoscopy $1991 ; 23$ : 305-306.

Corresponding Author

J. Pérez-Piqueras, M.D., Servicio de Aparato Digestivo, Hospital Universitario del Aire, Calle Arturo Soria 82, 28027 Madrid, Spain 